OPEN ACCESS

Edited by:

Gemmy Cheung,

Singapore National Eye

Center, Singapore

Reviewed by:

Min Sagong,

Yeungnam University, South Korea

Jurgen Sota,

University of Siena, Italy

*Correspondence:

Panpan Ye

yepanpan@zju.edu.cn

${ }^{\dagger}$ These authors have contributed equally to this work

Specialty section: This article was submitted to

Ophthalmology,

a section of the journa

Frontiers in Medicine

Received: 16 December 2020 Accepted: 22 March 2021

Published: 29 April 2021

Citation:

Xu Y, Shan Y, Hu Y, Cao J, Wang Y, Lou L and Ye P (2021) Case Report:

An Adult Patient With Deficiency of

Adenosine Deaminase 2 Resembled

Unilateral Frosted Branch Anguitis.

Front. Med. 8:642454.

doi: 10.3389/fmed.2021.642454

\section{Case Report: An Adult Patient With Deficiency of Adenosine Deaminase 2 Resembled Unilateral Frosted Branch Angiitis}

\author{
Yufeng $\mathrm{Xu}^{1+}$, Yi Shan ${ }^{1 \dagger}$, Yin $\mathrm{Hu}^{2 \dagger}$, Jing Cao ${ }^{1}$, Yijie Wang ${ }^{1}$, Lixia Lou ${ }^{1}$ and Panpan Ye ${ }^{1 *}$ \\ 'Eye Center, College of Medicine, The Second Affiliated Hospital of Zhejiang University, Zhejiang, China, ${ }^{2}$ Department of \\ Neurology, College of Medicine, The Second Affiliated Hospital of Zhejiang University, Zhejiang, China
}

Purpose: Deficiency of adenosine deaminase 2 (DADA2) is a rare autosomal recessive systemic autoinflammatory disorder. We describe a rare case of an adult patient with DADA2 who presented with unilateral frosted branch angiitis (FBA) combined with branch retinal vein occlusion and panuveitis.

Method: This paper is a clinical case report.

Results: A 31-year-old male patient complained of blurred vision in his right eye for 2 days. His fundus examination showed FBA combined with branch retinal vein occlusion and panuveitis. He had a medical history of intermittent and recurrent fever, skin rash and aphthous ulcer for 5 years, and lacunar infarction for 1 month. Laboratory examinations showed hypogammaglobulinemia and mild prolonged activated partial thromboplastin time (APTT). Brain magnetic resonance imaging (MRI) revealed old lacunar infarction in the right basal ganglia and the lateral ventricle and fresh lacunar infarction in the right pons, respectively. The perivascular sheathing of FBA and macular edema were resolved after steroid administration and treatment of intravitreal anti-VEGF injection. During the period of follow-up, the patient subsequently suffered from recurrence of strokes, abnormality of coagulation function, sudden hearing loss of the left ear, and diplopia. His gene sequencing results demonstrated several deletion mutations in ADA2, and the diagnosis of DADA2 was eventually confirmed.

Conclusions: FBA represents a very rare ocular feature of DADA2 and may in some cases be the presenting manifestation. Therefore, ophthalmologists need to be aware of this rare autoinflammatory disease.

Keywords: deficiency of adenosine deaminase 2, frosted branch angiitis, autoinflammatory and autoimmune diseases, retina vascular disorder, blood coagulation factor XII

\section{INTRODUCTION}

Frosted branch angiitis (FBA), a rare form of a vascular disorder, was first report on an immunocompetent 6-year-old Japanese boy in 1976 (1). Subsequent reports delineated the typical findings: acute visual loss, variable inflammation with perivascular sheathing, late staining of vessels usually without obstruction of blood flow, and a rapid response to systemic corticosteroids 
(2). In recent years, several unusual FBA cases associated with ophthalmic and systemic autoimmune/autoinflammatory disorders [e.g., Crohn's disease (3), Behcet's disease (4), and systemic lupus erythematosus (5)] were reported. Deficiency of adenosine deaminase 2 (DADA2) is a systemic autoinflammatory disorder caused by a loss-of-function (LOF) mutation in the cat eye syndrome critical region protein 1 (CECR1, also called $A D A 2)$ gene, of which the three major manifestations are vasculitis, immunologic manifestations, as well as hematologic features. Its major symptoms usually appear in early childhood (i.e., age $<10$ years) $(6-8)$. Here, we present the first case of FBA-like vasculitis in an adult DADA2 patient who also carries a heterozygous mutation of blood coagulation factor XII (F XII).

\section{CASE REPORT}

A 31-year-old Chinese male with sudden onset of blurred vision in his right eye for 2 days was referred to our clinic on January 21, 2019. On examination, best corrected visual acuity (BCVA) was 20/20 and 20/1,000 in the left and right eye, respectively. Furthermore, intraocular pressure (IOP) was elevated to $39.3 \mathrm{mmHg}$ in the right eye. A slit lamp demonstrated ciliary hyperemia, positive fresh keratic precipitates (KP), positive anterior chamber flare and cell, and positive relativistic afferent pupillary disorder (RAPD) in the right eye. On fundus examination, the right eye showed prominent white sheathing of retinal venules and, to a lesser extent, arterioles in all the quadrants, resembling the appearance characterized as "frosted branch angiitis." Supratemporal retinal hemorrhages indicated concomitant branch retinal vein occlusion (BRVO). Moreover, macular edema and disc hyperemia were presented (Figure 1A). Fundus photograph of the left eye showed normal appearance (Figure 1B). Optical coherence tomography (OCT) suggested macular edema (Figure 1D). Fluorescein angiography (FA) revealed supratemporal bleeding-blocked fluorescence, concomitant capillary non-perfusion zone, and extensive dye leakage from vessels and the optic disc (Figures 1G,H). Positive anterior chamber flare and cells, together with retinal exudation and macular edema, also indicated panuveitis.

The patient has a history of intermittent and recurrent fever, skin rash (Figure 1C), and aphthous ulcer from 5 years ago. Tests from other hospitals implicated hypogammaglobulinemia with immune globulin (Ig) G $4.520 \mathrm{~g} / \mathrm{l}$, IgA $4.632 \mathrm{~g} / \mathrm{l}$, and IgM 0.134 $\mathrm{g} / \mathrm{l}$. One month ago, the patient visited a neurology clinic with complaints of dizziness, nausea, vomiting, totter, and slurred speech. Magnetic resonance imaging (MRI) revealed lacunar infarction in the right basal ganglia and the lateral ventricle (Figure 1E). Activated partial thromboplastin time (APTT) was mildly prolonged to $50.3 \mathrm{~s}$. CBC showed elevated neutrophil percentage (NEU\%) of $88.1 \%$ and decreased lymphocyte (LYM\#) of $0.45 \times 10^{9} / \mathrm{L}$. Hepatorenal function, cranial angiography, carotid and cardiac ultrasound, and dynamic electrocardiogram were uneventful. Because of self-claimed allergy to aspirin, patient was administered clopidogrel and atorvastatin once a day.
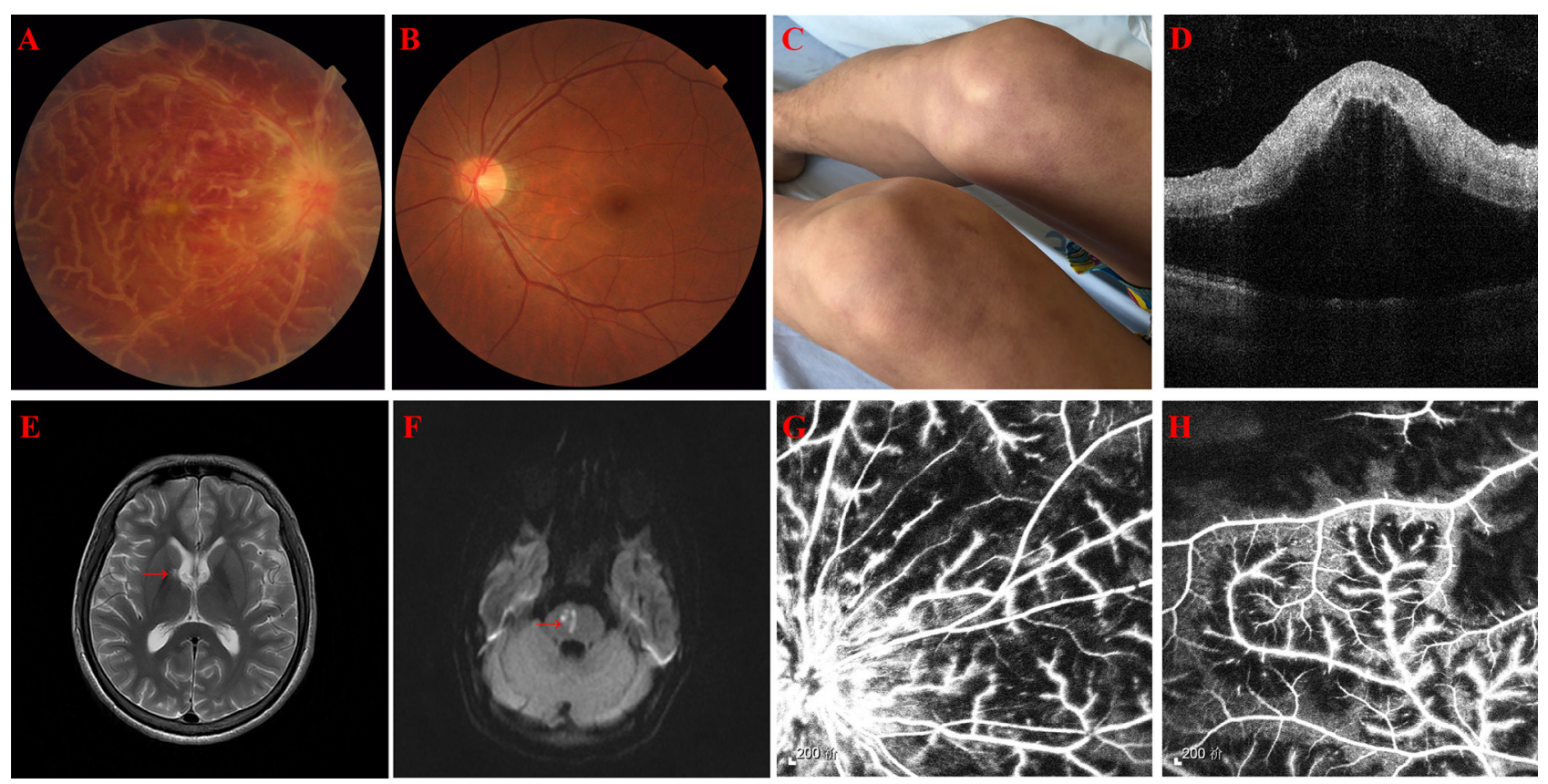

FIGURE 1 | (A) A fundus photograph of the right eye showing thick, white confluent sheathing around retinal veins suggestive of frosted branch angiitis, disc hyperemia, and macular edema. (B) A fundus photograph of the left eye showing normal appearance. (C) Recurrent skin rash on both thighs. (D) Optical coherence tomography of the right eye showing exudative macular detachment, vitreous cells, and posterior vitreous detachment. (E) Old lacunar infarction involving the right basal ganglia and the lateral ventricle (red arrow) in magnetic resonance (MR) T2 phase, but not in the diffusion-weighted imaging (DWI) (image not showed). (F) Fresh lacunar infarction in the right pons (red arrow) in MR DWI. (G,H) Fluorescein angiography of the right eye showing extensive vascular leakage and supratemporal capillary non-perfusion areas. 

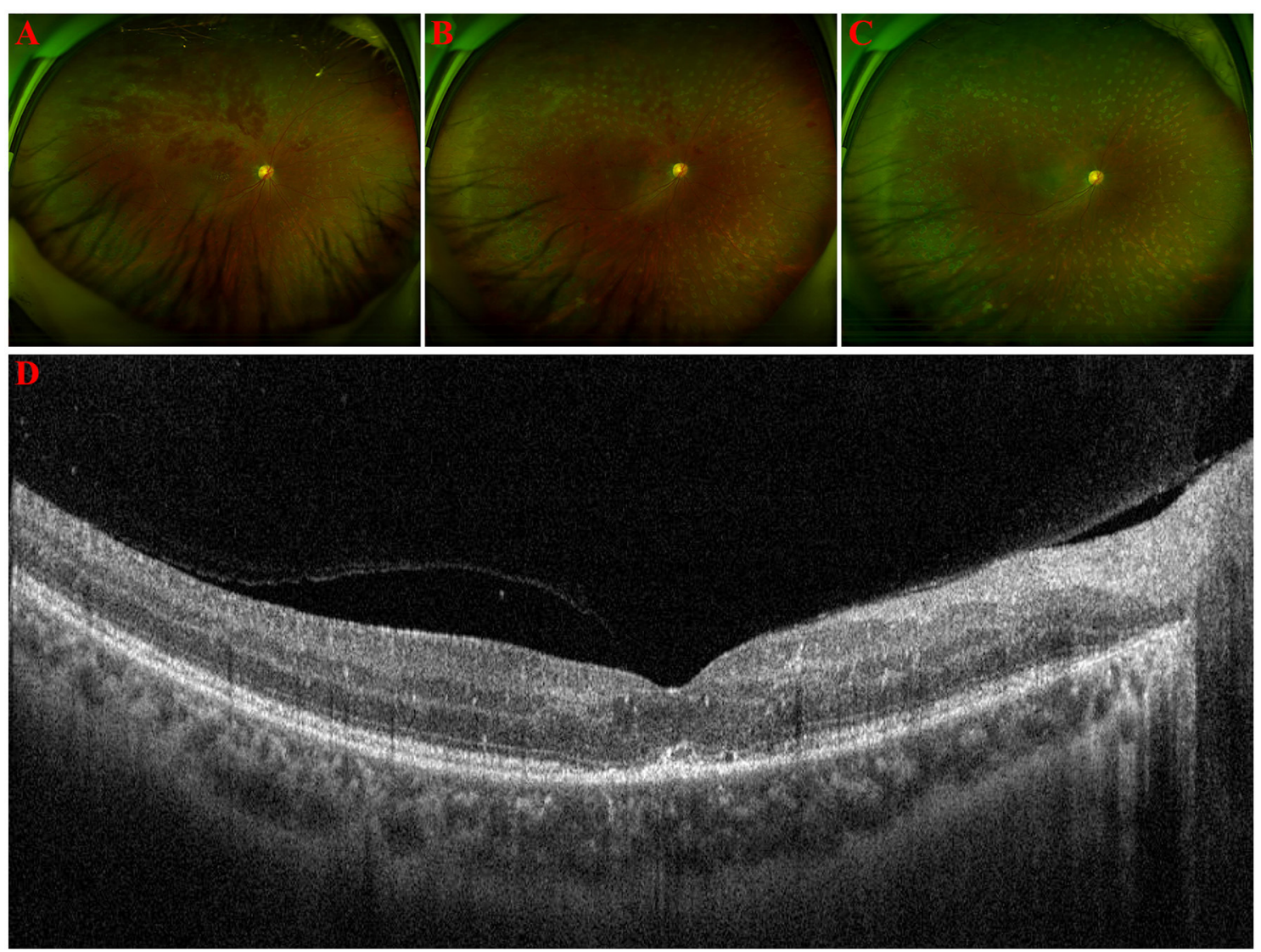

FIGURE 2 | (A-C) Ultra-wide-angle fundus photographs of the right eye from 1, 2, and 5 months post-treatment. White sheathing of the vessels alleviated first, and supratemporal hemorrhage resolved at 5 months later. (D) Optical coherence tomography of the right eye 1 month after treatment showing disappearance of subretinal exudation and much less vitreous cells.

With consultation from neurologists and rheumatologists, the following laboratory investigations were carried out: raised $\mathrm{C}$ reactive protein (CRP) of $41.2 \mathrm{mg} / \mathrm{l}$, homocysteine of $16.0 \mu \mathrm{mol} / \mathrm{l}$, decreased CD25 + lymphocyte of $4.06 \%$, helper T-cell of $3.70 \%$, IgG of $4.3 \mathrm{~g} / \mathrm{l}$, IgA of $0.48 \mathrm{~g} / \mathrm{l}$, and IgM of $<0.25 \mathrm{~g} / \mathrm{l}$, and microcytic hypochromic anemia was noticed. Other workups, including urine routines, erythrocyte sedimentation rate (ESR), coagulation function, streptolysin $\mathrm{O}$, rheumatoid factor, antiphospholipid antibodies, antineutrophilic cytoplasmic antibodies, antinuclear antibodies, protein $\mathrm{C}$, protein S, Toxoplasma gondii, rubella, herpes simplex, hepatitis B, hepatitis C, Epstein-Barr virus, HIV, cytomegalovirus, Treponema pallidum, and Mycobacterium tuberculosis, were negative. Anterior chamber paracentesis for high-throughput sequencing did not find any pathogenic microorganisms.

Oral methylprednisolone $24 \mathrm{mg}$ /day was started and tapered off within 3 months, together with three times of periocular injection of $20 \mathrm{mg}$ methylprednisolone. Topical fluorometholone $(0.1 \%)$ and pranoprofen $(0.1 \%)$ eye drops four times per day were administered. Carteolol (2\%) and brinzolamide (1\%) eye drops were used to alleviate IOP. Focal retinal photocoagulation and intravitreal injection of ranibizumab $(0.5 \mathrm{mg} / 50 \mu \mathrm{l})$ were given to control fundus hemorrhage. One week after treatment, KP and aqueous flare and cells disappeared and the IOP decreased back to normal. One month later, the macular edema was resolved in the right eye (Figure 2D). White sheathing of the vessels alleviated (Figure 2A). Two months later, a fundus photo showed full resolution of vascular sheathing (Figure 2B) and visual acuity improved to 20/133. Retinal hemorrhage was absorbed at 5 months follow-up (Figure 2C).

However, the patient came to our emergency room (ER) with a complaint of left limb weakness and deterioration of slurred speech for 3 days, 5 months after being diagnosed of FBA. APTT was prolonged by $47.8 \mathrm{~s}, \mathrm{~F}$ XI and F XII activity was decreased to $66.7 \%$ (reference range: $70-120 \%$ ) and $6.6 \%$ (reference range: $70-150 \%)$, respectively. MRI confirmed fresh lacunar infarction in the right pons (Figure 1F). The patient was diagnosed with patent foramen ovale (PFO) by contrast echocardiography of the right heart during admission and underwent a catheterbased closure surgery then. One month later, our patient was admitted in the emergency department of another center, complaining aggravation of left limb weakness and slurred speech. Due to the recent cardiac surgical implantation, cranial computerized tomography (CT) was adopted instead 


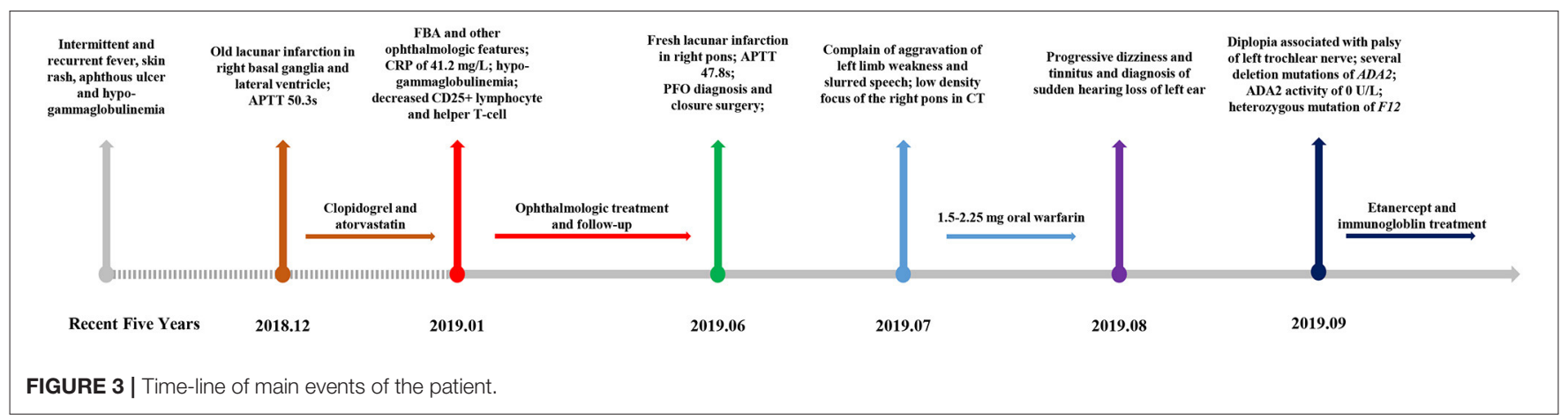

of MRI, which implicated low-density focus of the right pons. Considering deteriorating strokes under clopidogrel and atorvastatin intervention, $1.5-2.25 \mathrm{mg}$ oral warfarin was given to prevent cryptogenic stroke, with international normalized ratio (INR) maintained between 2 and 3. One month later, the patient appeared with progressive dizziness and tinnitus and was diagnosed of sudden hearing loss of the left ear. Then, the patient presented with diplopia associated with palsy of the left trochlear nerve. Given the patient's complicated multiple disorders, whole exome sequencing (WES) was adopted to explore the possible pathogenesis. Several deletion mutations of ADA2 (homozygous deletion of exon 7 and heterozygous deletion of exons 2-6 and 8-10) and heterozygous mutation of F12 (p.K365Qfs*69) were recognized. Subsequent laboratory test demonstrated a total ADA activity of $2.2 \mathrm{U} / 1$ and an ADA2 activity of $0 \mathrm{U} / \mathrm{l}$. Anti-TNF therapy was then started by weekly injection of $50 \mathrm{mg}$ etanercept. Meanwhile, the patient received intravenous immunoglobulin according to monitoring. The brief timeline of our case is described in Figure 3.

\section{DISCUSSION}

FBA is a rare form of vasculitis characterized by florid translucent retinal perivascular sheathing, resembling the appearance of frosted tree branches in winter. Classical symptoms include sudden onset of blurred vision with floaters and photopsia. Despite the absence of a standard guideline treatment, most patients respond well with systemic steroids and have rapidly resolved with good visual recovery. Recurrence is rare. However, the prognosis has not always been favorable. Common complications include retinal vein/artery occlusion, fundus hemorrhage, optic disc atrophy, and macular edema (2).

The cause of FBA is unknown. Kleiner classified the patients with similar FBA appearance into three subgroups (9). First are the patients with lymphoma (10) or leukemia (11) whose disease is likely due to malignant cell infiltration. The second group consists of patients who have associated infections [including tuberculosis (12), syphilis (13), cytomegalovirus (14), HIV (15), rubella (16), Epstein-Barr virus (17), herpes simplex virus (18), etc.] or autoimmune diseases [such as Crohn's disease (3), Behcet's disease (4), systemic lupus erythematosus (5), etc.]. Inflammatory processes like vasculitis, immune complex deposition, or hypersensitivity reactions to microorganisms are considered to be the underlying mechanisms. The third group is composed of otherwise healthy young patients, who are probably undergoing immune response to underlying stimulus such as a viral infection. Nevertheless, until possible causative agents can be identified, the similar clinical appearances and courses were referred to as "acute idiopathic frosted branch angiitis."

DADA2 is a systemic autoinflammatory disorder caused by LOF mutation in the ADA2 gene, of which the three major manifestations are vasculitis, dysregulation of immune function, and hematologic disease (6-8). Inflammatory features include intermittent fevers, rash (often livedo racemosa/reticularis), and cutaneous ulcer. Vasculitis may manifest as early onset and recurrence of ischemic (lacunar) and/or hemorrhagic strokes or as cutaneous or systemic polyarteritis nodosa. Dysregulation of immune function can result in immunodeficiency or autoimmunity of varying severity. Hematologic disorders may begin early in life or rarely in late adulthood and can include lymphopenia, neutropenia, pure red cell aplasia, thrombocytopenia, or pancytopenia. Other clinical findings consist of musculoskeletal features, aphthous ulcers, inflammatory bowel disease-like illness, and hearing loss (19). To be noted, some manifestations of DADA2 might often overlap with polyarteritis nodosa (PAN), while others are more unique, especially immune deficiency and hematologic disease. Many clinical features or vessel histopathology do not appear to be helpful in differentiating DADA2 from PAN. Though there is a lack of guideline, gene and ADA2 functional evaluation is recommended in the diagnosis of DADA2 $(19,20)$.

Ophthalmologic manifestations have been noticed since DADA2 was first defined. Zhou et al. (21) reported ophthalmologic manifestations in five patients (total nine patients), including central retinal artery occlusion (CRAO) in one patient, optic nerve atrophy in one, diplopia with irregular enhancement of the medial rectus muscle (as indicated by cranial MRI) in one, third cranial nerve palsy in one, and strabismus in two. Furthermore, patients could have more than one ophthalmologic disorder. More recently, Sahin et al. (22) revealed ocular symptoms/signs in four patients (total eight patients), including temporary monocular/binocular vision loss in two patients, strabismus in two, panuveitis in one, and CRAO in one. These ophthalmologic features were likely to be contributed by possible central retinal artery involvement 
and third cranial nerve palsies after small initially undetectable strokes. Ocular inflammation (uveitis, scleritis, and episcleritis) (23), ptosis, and nystagmus were also documented $(19,24)$. So far, there have not been reports about a DADA2 patient displaying retinal vasculitis resembling FBA.

Experiments indicated that cecrlb is essential for both vascular integrity and neutrophil development in the zebrafish embryo and that both phenotypes are prevented by non-mutant, but not by mutant, human CECR1 mRNA (21). DADA2 may compromise endothelial integrity while polarizing macrophage and monocyte subsets toward proinflammatory cells, establishing a vicious circle of vasculopathy and inflammation $(25,26)$, which is likely to trigger infiltration and exudation of retinal vessel presenting an FBA appearance. Our patient suffered from intermittent and recurrent fever, skin rash, lacunar infarctions, hypogammaglobulinemia, and aphthous ulcer and presented retinal vasculitis with hemorrhage, sudden hearing loss, and diplopia. A subsequent test confirmed deletion of several ADA2 exons with undetectable activity of ADA2 enzyme activity. Though no defined treatment strategies exist, anti-TNF agents (including etanercept, adalimumab, golimumab, infliximab, and certolizumab) have presented a remarkable effect on preventing ADA2-associated vasculitis (especially in reducing the risk of stroke) and, in some degree, relieving inflammatory burden. Hematopoietic stem cell transplantation (HSCT) can be curative to severe bone marrow abnormalities, which have poor response to anti-TNF agents. Steroids and general immunosuppressive therapies also have had variable success (27). In our case, the patient responded well to oral and topical administration of steroid without recurrence of perivascular sheathing and gained significant improvement of vision acuity. Elevated IOP and concomitant BRVO were also alleviated with the additional help of retinal photocoagulation, topical hypotensive, and antiVEGF agents.

This patient also carried a heterozygous mutation of blood coagulation factor XII (F XII), which may lead to deficiency of F XII resulting in the development of thromboembolism rather than bleeding complications. A few investigators found that deficiency of F XII might be in association with elevated risk of retinal vessel occlusion $(28,29)$. However, other researchers hold a complete opposite opinion that mild F XII deficiency neither causes thrombosis nor protects from thrombosis (30-33).

\section{REFERENCES}

1. Ito Y, Nakano M, Kyu N, Takeuchi M. Frosted branch angiitis in a child. Jpn J Clin Ophthalmol. (1976) 30:797-803.

2. Walker S, Iguchi A, Jones NP. Frosted branch angiitis: a review. Eye (Lond). (2004) 18:527-33. doi: 10.1038/sj.eye.6700712

3. Sykes SO, Horton JC. Steroid-responsive retinal vasculitis with a frosted branch appearance in Crohns disease. Retina. (1997) 17:451-4. doi: 10.1097/00006982-199717050-00017

4. Ferreira BFA, Rodriguez EEC, Prado LLD, Goncalves CR, Hirata CE, Yamamoto JH. Frosted branch angiitis and cerebral venous sinus thrombosis as an initial onset of neuro-Behcet's disease: a case report and review of the literature. J Med Case Rep. (2017) 11:104. doi: 10.1186/s13256-0171261-z
Based on current evidence and research basis, we could not ascertain whether the heterozygous mutation of F XII condition in our patient contributed to retinal vasculitis and other systemic symptoms or not. However, considering the heterozygous mutation of F XII and only mild prolonged APTT, it was not likely to play a vital role in this case.

To our best knowledge, this is the first case report of a patient with FBA-like retinal vasculitis due to adult DADA2. Although ADA2 is not expressed in endothelial cells, there is a defect in endothelial integrity in the small vessels of patients with ADA2 mutations as well as an impairment of M2 macrophage differentiation (34), which might cause the FBA appearance. In summary, FBA can be a presenting feature in DADA2 disorder, of which ophthalmologists need to be aware. Prompt treatment with corticosteroids and anti-inflammatory agents can lead to good remission.

\section{DATA AVAILABILITY STATEMENT}

The original contributions presented in the study are included in the article/supplementary material, further inquiries can be directed to the corresponding author/s.

\section{ETHICS STATEMENT}

The studies involving human participants were reviewed and approved by The Second Affiliated Hospital of Zhejiang University School of Medicine Review Board and Ethics Committee (No. 2020-286). The patients/participants provided their written informed consent to participate in this study.

\section{AUTHOR CONTRIBUTIONS}

All authors listed have made a substantial, direct, and intellectual contribution to the work, and approved it for publication.

\section{FUNDING}

This study was supported by the Natural Science Foundation of China (81900853) and the China Postdoctoral Science Fund (2019M652107). 
9. Kleiner RC. Frosted branch angiitis: clinical syndrome or clinical sign? Retina. (1997) 17:370-1. doi: 10.1097/00006982-199709000-00002

10. Ridley ME, McDonald HR, Sternberg P, Jr., Blumenkranz MS, Zarbin MA, Schachat AP. Retinal manifestations of ocular lymphoma (reticulum cell sarcoma). Ophthalmology. (1992) 99:1153-60; discussion 1160-1. doi: 10.1016/S0161-6420(92)31834-2

11. Kim TS, Duker JS, Hedges TR. 3rd. Retinal angiopathy resembling unilateral frosted branch angiitis in a patient with relapsing acute lymphoblastic leukemia. Am J Ophthalmol. (1994) 117:806-8. doi: 10.1016/S0002-9394(14)70329-0

12. Fountain JA, Werner RB. Tuberculous retinal vasculitis. Retina. (1984) 4:4850. doi: 10.1097/00006982-198400410-00008

13. Lobes LA, Jr., Folk JC. Syphilitic phlebitis simulating branch vein occlusion. Ann Ophthalmol. (1981) 13:825-7.

14. Mao F, Wu J, Sun H, You Q, Li D. Frosted branch angiitis in an AIDS patient with cytomegalovirus retinitis. Int J Infect Dis. (2016) 52:9-11. doi: 10.1016/j.ijid.2016.09.003

15. Fine HF, Smith JA, Murante BL, Nussenblatt RB, Robinson MR. Frosted branch angiitis in a child with HIV infection. Am J Ophthalmol. (2001) 131:394-6. doi: 10.1016/S0002-9394(00)00792-3

16. Biswas J, Fogla R, Madhavan HN. Bilateral frosted branch angitis in an 8-year-old Indian girl. Retina. (1996) 16:444-5. doi: 10.1097/00006982-199616050-00014

17. Farrando J, Fonollosa A, Segura A, Garcia-Arumi J. Frosted branch angiitis associated with Epstein-Barr virus systemic infection. Ocul Immunol Inflamm. (2008) 16:41-3. doi: 10.1080/09273940701799114

18. Barkmeier AJ, Feman SS. Frosted branch angiitis secondary to herpes simplex virus infection progressing to acute retinal necrosis. Retin Cases Brief Rep. (2009) 3:36-7. doi: 10.1097/ICB.0b013e318159e825

19. Aksentijevich I, Sampaio Moura N, Barron K. Adenosine Deaminase 2 Deficiency BTI - GeneReviews((R)). In: GeneReviews ${ }^{\circledR}$ [Internet]. Seattle, WA: University of Washington (2019).

20. Human A, Pagnoux C. Diagnosis and management of ADA2 deficient polyarteritis nodosa. Int $J$ Rheum Dis. (2019) 22(Suppl 1):69-77. doi: 10.1111/1756-185X.13283

21. Zhou Q, Yang D, Ombrello AK, Zavialov AV, Toro C, Zavialov AV, et al. Earlyonset stroke and vasculopathy associated with mutations in ADA2. $\mathrm{N} \mathrm{Engl} \mathrm{J}$ Med. (2014) 370:911-20. doi: 10.1056/NEJMoa1307361

22. Sahin S, Adrovic A, Barut K, Ugurlu S, Turanli ET, Ozdogan H, et al. Clinical, imaging and genotypical features of three deceased and five surviving cases with ADA2 deficiency. Rheumatology Int. (2017) 38:129-36. doi: 10.1007/s00296-017-3740-3

23. Gibson KM, Morishita KA, Dancey P, Moorehead P, Drogemoller B, Han $\mathrm{X}$, et al. Identification of novel adenosine deaminase 2 gene variants and varied clinical phenotype in pediatric vasculitis. Arthritis Rheumatol. (2019) 71:1747-55. doi: 10.1002/art.40913

24. Tanatar A, Karadag SG, Sozeri B, Sonmez HE, Cakan M, Kendir Demirkol $\mathrm{Y}$, et al. ADA2 Deficiency: case series of five patients with varying phenotypes. J Clin Immunol. (2019) 40:253-58. doi: 10.1007/s10875-019-0 0734-0

25. Moore KJ, Tabas I. Macrophages in the pathogenesis of atherosclerosis. Cell. (2011) 145:341-55. doi: 10.1016/j.cell.2011.04.005

26. Woollard KJ, Geissmann F. Monocytes in atherosclerosis: subsets and functions. Nat Rev Cardiol. (2010) 7:77-86. doi: 10.1038/nrcardio.2009.228

27. Ombrello AK, Qin J, Hoffmann PM, Kumar P, Stone D, Jones A, et al. Treatment Strategies for Deficiency of Adenosine Deaminase 2. N Engl J Med. (2019) 380:1582-4. doi: 10.1056/NEJMc1801927

28. Kuhli C, Scharrer I, Koch F, Ohrloff C, Hattenbach L-O. Factor XII deficiency: a thrombophilic risk factor for retinal vein occlusion. Am J Ophthalmol. (2004) 137:459-64. doi: 10.1016/j.ajo.2003.10.024

29. Abu el-Asrar AM, al-Momen AK, al-Amro S, Abdel Gader AG, Tabbara KF. Prothrombotic states associated with retinal venous occlusion in young adults. Int Ophthalmol. (1996) 20:197-204. doi: 10.1007/BF00175260

30. Koster T, Rosendaal FR, Briet E, Vandenbroucke JP. John Hageman's factor and deep-vein thrombosis: Leiden thrombophilia Study. Br J Haematol. (1994) 87:422-4. doi: 10.1111/j.1365-2141.1994.tb04937.x

31. Zeerleder S, Schloesser M, Redondo M, Wuillemin WA, Engel W, Furlan $\mathrm{M}$, et al. Reevaluation of the incidence of thromboembolic complications in congenital factor XII deficiency-a study on 73 subjects from 14 Swiss families. Thromb Haemost. (1999) 82:1240-6. doi: 10.1055/s-0037-16 14368

32. Girolami A, Randi ML, Gavasso S, Lombardi AM, Spiezia F. The occasional venous thromboses seen in patients with severe (homozygous) FXII deficiency are probably due to associated risk factors: a study of prevalence in 21 patients and review of the literature. J Thromb Thrombolysis. (2004) 17:139-43. doi: 10.1023/B:THRO.0000037670.42776.cd

33. Nakamura A, Osonoi T, Terauchi Y. Relationship between urinary sodium excretion and pioglitazone-induced edema. J Diabetes Investig. (2010) 1:20811. doi: 10.1111/j.2040-1124.2010.00046.x

34. Zavialov AV, Gracia E, Glaichenhaus N, Franco R, Zavialov AV, Lauvau G. Human adenosine deaminase 2 induces differentiation of monocytes into macrophages and stimulates proliferation of $\mathrm{T}$ helper cells and macrophages. J Leukoc Biol. (2010) 88:279-90. doi: 10.1189/jlb.11 09764

Conflict of Interest: The authors declare that the research was conducted in the absence of any commercial or financial relationships that could be construed as a potential conflict of interest.

Copyright (c) $2021 \mathrm{Xu}$, Shan, Hu, Cao, Wang, Lou and Ye. This is an open-access article distributed under the terms of the Creative Commons Attribution License (CC $B Y)$. The use, distribution or reproduction in other forums is permitted, provided the original author(s) and the copyright owner(s) are credited and that the original publication in this journal is cited, in accordance with accepted academic practice. No use, distribution or reproduction is permitted which does not comply with these terms. 